\title{
Fracture strength for metal structure of a crane
}

\author{
Vladimir Mironov ${ }^{1,2}$, Olga Lukashuk ${ }^{2, *}$, and Dmitriy Ogorelkov² \\ ${ }^{1}$ Institute of Engineering Science, RAS (Ural Branch), Yekaterinburg, Russia \\ ${ }^{2}$ Ural Federal University, Yekaterinburg, Russia
}

\begin{abstract}
Anthropogenic catastrophes, as a rule, are caused directly by severe violations of operating rules set for technical systems and devices. One accident is considered in the article, where the rotating section of a tower crane fell down. A conjecture based on calculations is proposed as to what could have possibly caused the fracture of a bolted joint between the slewing unit and mast shell.
\end{abstract}

\section{Introduction}

The problem of fracture strength of metal structures is topical in many fields of technology. And especially so in the engineering of lifting and carrying machines, where, along with the demand for lower metal intensity and power inputs, excessive requirements are set for product safety [1-4]. Therefore, it is reasonable to supplement typical calculations made for nominal loads with those for bearing capacity of a structure under extreme conditions when fractured elements start to appear.

Excessive loads could be caused by a fall of lifted load, weather cataclysms, severe violations of operating rules, etc. As a rule, such loads are dynamic by their nature and often lead to fracture of a structure, material losses and human deaths. The goal of auxiliary calculations in such a case is to determine ultimate loads and check the strength of the structure.

As a rule, the emergence of anthropogenic hazards is related to various discrepancies appearing due to failure to comply with different requirements. The article analyzes the situation which happened with a tower crane POTAIN MD 265 B J12 operating at a regular angular velocity and without a load. The analysis of operating conditions and the pattern of crane fracture has revealed several major discrepancies: fatigue cracks in the shell under the rotating section (Fig. 1, a), a fullcircle trail where the rotating section were touching the base (Fig. 1, b), dilapidated teeth of the slewing ring (Fig. 1, c). One could not have missed any discrepancy from that list during day-by-day visual examination. Jamming of the slewing mechanism could be caused by any factor mentioned above.

According to the ISO 9001 standard [1], when a person in charge of crane operation learns about any discrepancy, he must initiate the correction procedure to determine the causes of the violation and eliminate them.
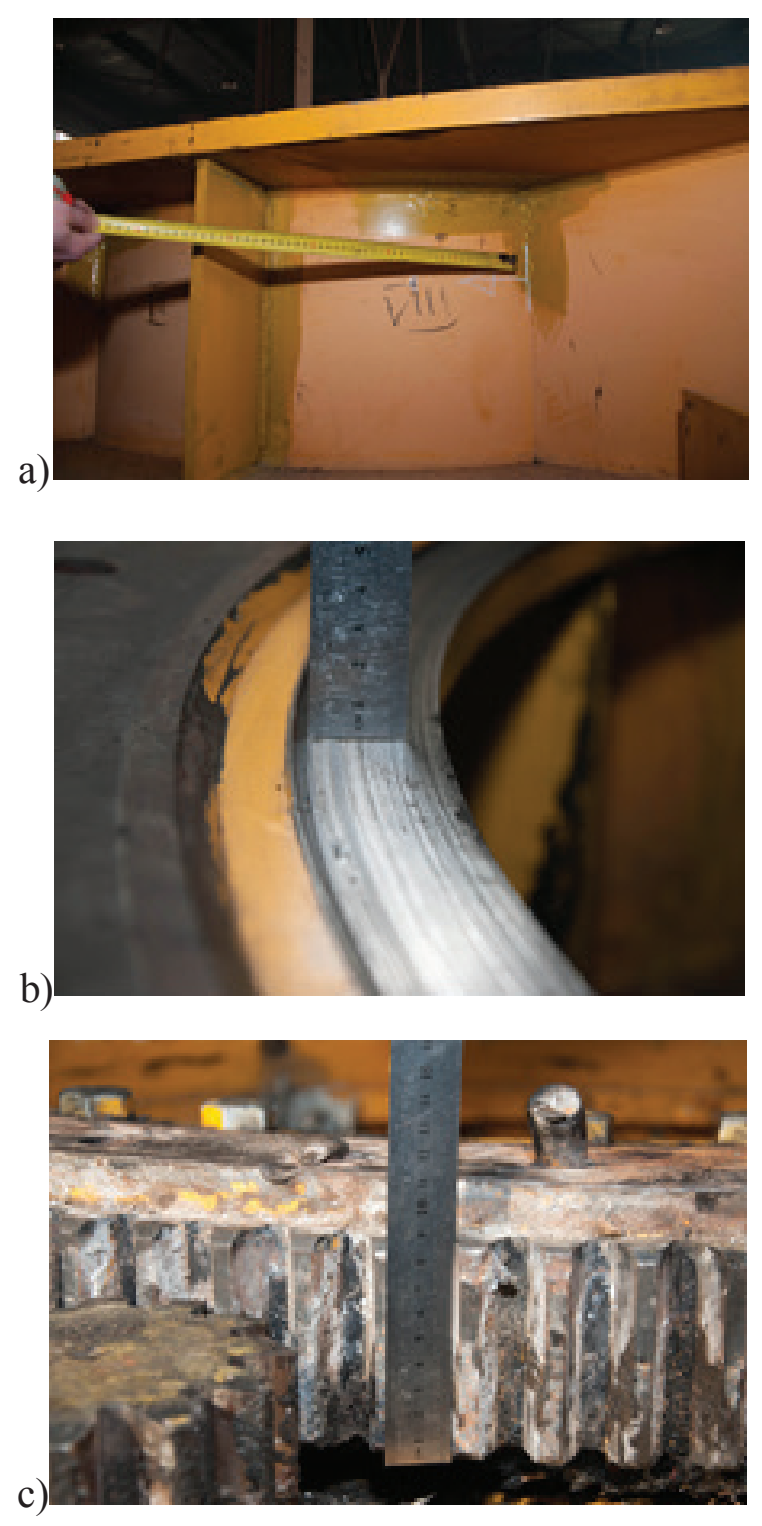

Fig. 1. a) welded cracks, b) full-circle trail, c) slewing ring.

\footnotetext{
* Corresponding author: oldim96@mail.ru
} 
The shell body shows signs of welded cracks, thus indicating the correction which was made. But there is no guarantee of its helping to maintain normal conditions for the operation of balls in the slewing unit. The second and third discrepancies could have been caused by the repair work, which was introduced by means welding open fatigue cracks, and led to wedging of the slewing mechanism. Other factors might have played a role in jamming, like, loose bolts by which the fixed part of the slewing unit was fastened to the shell or incomplete set of those.

It is supposed that bolted flange joint of the rotating section was fractured due to wedging of the slewing unit. Notwithstanding the real reasons behind jamming of the slewing mechanism, it is necessary to find out whether or not it could be the cause of such an accident. The goal of the work is to determine dynamic loads when wedging of a slewing mechanism occurs and check the strength of bolts similar to the ones fractured during the accident. To check the working hypothesis on what caused the rotating section of the crane to fall, the following tasks were solved:

- dynamic models were built, and peak forces determined, for the motions of the jib and counterweight (plus its frame), all considered as reduced localized masses;

- severing reduced forces, bending and torsion moments applied to bolting joints were evaluated;

- a group bolted joint was evaluated, with equivalent stresses determined and pie chart of their distribution among bolts of the joint plotted;

- finite element analysis for the counterweight frame was carried out to check the rigidity of the structure and establish whether or not it is expedient to reinforce the bolted joint unit;

- the conclusion was made on what could have possibly caused the rotating section to fall, and one proposed variant of engineering changes was considered.

\section{Jib and counterweight vibrations after slewing unit wedging}

Fig. 2 shows schematics of the crane rotating section. A lifting jib of $L_{J}=65 \mathrm{~m}$ in length and a counter-jib of $L_{C}=18 \mathrm{~m}$ in length are fastened by means of flapping hinges to a column of $h=2,4 \mathrm{~m}$ in height and supported by guy lines. The accident occurred when the crane was rotating at an angular velocity of $\omega=0,08378 \mathrm{rad} / \mathrm{s}$ and with no load on its hook. A 12,83-ton mass of the lifting jib is reduced to its end, and $Q_{J R}=31,5 \mathrm{kN}$. A 9,83-ton mass of the counter jib is reduced to the load, and, with a 18-ton mass of the counterweight taken into account, $Q_{C}=200,7 \mathrm{kN}$. A circle diameter $d$ of the bolts which attach the base of the rotating section is equal to $1494 \mathrm{~mm}$. Elastic forces $F_{J}$ and $F_{C}$, which are to be determined, act in a horizontal plane and are caused by vibrations of the jib and counterweight which follow wedging of the slewing unit.

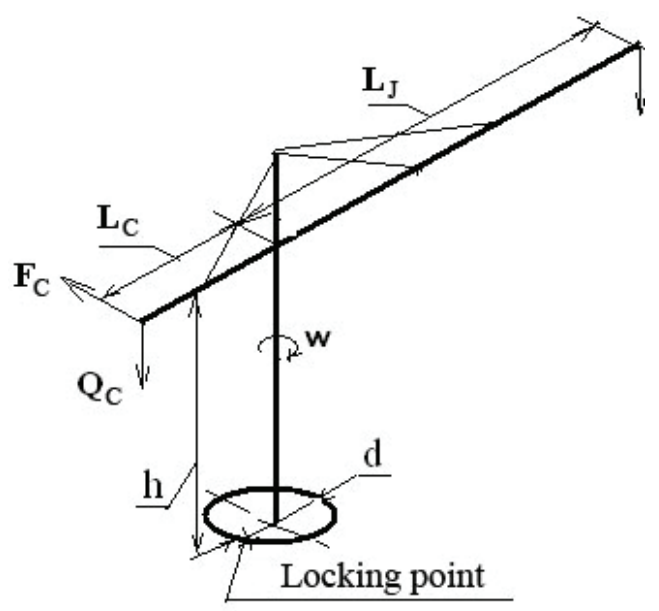

Fig. 2. Schematics of the crane rotating section.

Considering the trajectory curvature as negligible, let us select the scheme of translational motion for localized jib and counterweight masses: $m_{J}=3,2075 \mathrm{th}$ and $m_{C}=20,4587 \mathrm{tn}$.

Using D'Alembert's (kinetostatics) principle, we establish the equation of motion in the form of equilibrium condition for a mass $m$ and with inertia forces taken into account. It would be defined as following:

$$
m \dot{x}+c x=0 .
$$

Having defined an angular frequency as $p=\sqrt{c / m}$, we can reduce equation (1) to the canonical form of

$$
\dot{x}+p^{2} x=0 .
$$

The solution of a homogeneous differential equation with constant coefficients takes the form of [5]

$$
x=C_{1} \cdot \sin (p t)+C_{2} \cdot \cos (p t),
$$

where $C_{1}, C_{2}$ are integration constants determined on starting conditions. For $t=0: x_{0}=0, \dot{x}_{0}=\omega R$, where $R$ is the jib or counter-jib length.

The jib and counterweight have a similar pattern of motion, and specific parameters are determined by an initial speed and angular frequency

$$
x_{J}=\frac{\dot{x}_{0 J}}{p_{J}} \sin \left(p_{J} t\right), x_{C}=\frac{\dot{x}_{o C}}{p_{C}} \sin \left(p_{C} t\right) .
$$

The initial speed at the moment of wedging equals the linear motion speed of the jib end $\dot{x}_{0 J}$ and counterweight $\dot{x}_{0 C}$ :

$$
\dot{x}_{0 J}=\omega \cdot L_{J}=5,446 \mathrm{~m} / \mathrm{s}, \dot{x}_{0 C}=\omega \cdot L_{C}=1,508 \mathrm{~m} / \mathrm{s},
$$

where $\omega$ is the linear speed of the slewing mechanism.

In the first version of calculations, the rigidities of the jib and the counter-jib in a horizontal plane were determined by the formula (well-known from the 
strength of materials) for a girder restrained at one of its ends: $c=3 E J / L^{3}$.

The corresponding rigidities were

$$
c_{J}=3,0696 \mathrm{kN} / \mathrm{m} ; c_{C}=1381,6847 \mathrm{kN} / \mathrm{m},
$$

while angular frequencies $-p_{J}=0,9783 \mathrm{~s}^{-1}$ and $p_{C}=8,218 s^{-1}$, and periods of jib and counterweight vibrations $-T_{J}=6,42 \mathrm{~s}$ and $T_{C}=0,76 \mathrm{~s}$. Then, using the second formula in (2), we find the peak travel of the counterweight for $t_{*}=T_{C} / 4=0,19 \mathrm{~s}$, and, after that, the maximal force

$$
F_{C}=c_{C} \cdot x\left(t_{*}\right)=253,5 \mathrm{kN} .
$$

At that moment, the maximal force applied to the jib $F_{C}\left(t_{*}\right)=3,16 \mathrm{kN}$.

Then, force factors reduced to the plane in which severing of the bolts occurred were determined:

- bending moment in the vertical plane directed to the jib

$$
M_{1}=Q_{C} L_{C}-\mathrm{Q}_{J R} \mathrm{~L}_{J}=1565,1 \mathrm{kNm} ;
$$

- bending moment in the perpendicular plane

$$
M_{2}=\left(F_{C}-F_{J}\right) h=1051,4 \mathrm{kNm} ;
$$

- tension moment in the horizontal plane

$$
M_{T}=F_{C} L_{C}+F_{J} L_{J}=4357,6 \mathrm{kNm} ;
$$

- cross-sectional severing force

$$
Q_{\text {sev }}=F_{C}-F_{J}=250,34 \mathrm{kN} .
$$

\section{Calculation of bolted flange joint between slewing unit base and jib}

Assume that wedging of the mechanism occurs on the side of the counterweight close to bolt 24 (Fig. 3).

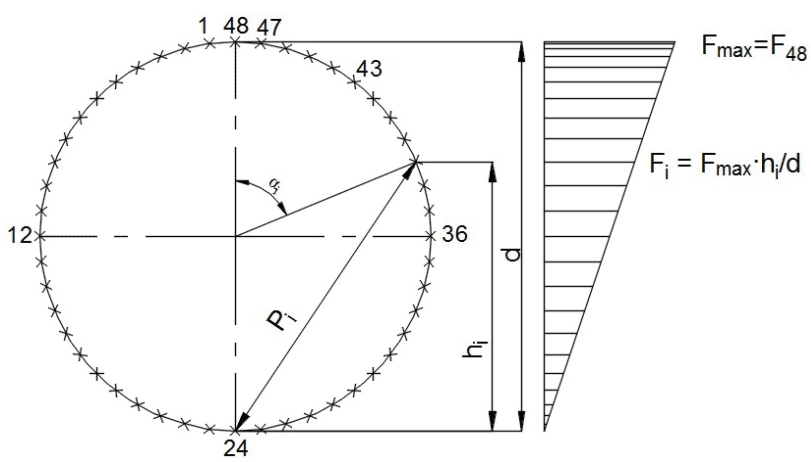

Fig. 3. Diagram of bolt locations and tensile strain distribution.

The direction $24-48$ is a trail of the plane of the moment $M_{1}$ action, and the direction $12-36$ is the same for the moment $M_{2}$. Suppose, as a common case, that a tensile strain in bolts $F_{i}$ is in direct proportion to a distance $h_{i}$. Then, on the condition of equality between the moments of external forces and the total moment of forces in bolts, tensile strains are determined for all the bolts in the group joint.

Next, normal stresses at the cross-section near the thread bottom along which the bolts broke during the accident were found. It was established that maximal calculated stress is in bolt 43: $\sigma_{43}\left(M_{1}, M_{2}\right)=341 M P a$. Since the joint had been loosened, tightening stresses and friction forces are neglected.

Then, it is necessary to allow for the concentration of stresses in the thread where fracture occurred (Fig. 4).

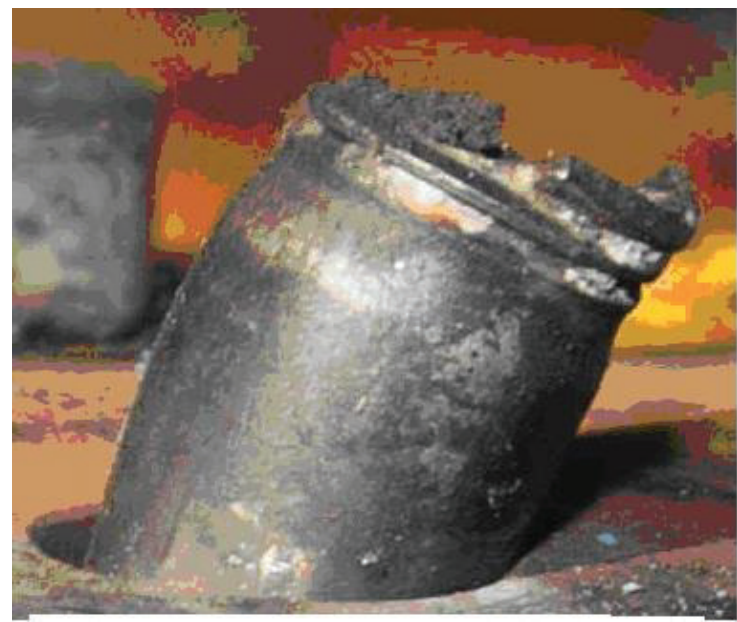

Fig. 4. Fractured bolt.

Bolts of 10.9 strength grade are made from highstrength steel with the yield point (yield stress) at $\sigma_{Y}=900 \mathrm{MPa}$ and the breaking point (ultimate stress) at $\sigma_{U}=1040 \mathrm{MPa}$. Concentration factor in the thread for such steels is estimated by different sources to be in the range $k \in[3,8-5,4]$, then, for $k=4$, the stress calculated for the most loaded bolt $\sigma_{C 43}=k \sigma_{43}\left(M_{1}, M_{2}\right)=1364 M P a$.

Such stress noticeably exceeds the ultimate stress for the bolt material and is somewhat higher than the dynamic yield stress $[6,7] \sigma_{Y D}=1,3 \sigma_{U}=1350 \mathrm{Mpa}$. But, to make a fair assessment of the bolt joint strength, it is necessary to allow for tangential severing stresses at the calculated cross-section along the groove bottom. The joint effect of normal and tangential stresses was evaluated on the fourth theory of strength

$$
\sigma_{e}=\sqrt{\sigma_{C}^{2}+3 \tau^{2}}
$$

Fig. 5 shows a circular diagram for equivalent stresses in the bolted joint for the maximal $F_{C}$. The stresses calculated for bolts $10 \div 32$ of the black zone (Fig. 5) exceed static yield stress of the material, and, for the zone of bolts $4 \div 38$, they are even higher than the dynamic yield stress. The bolt which turned out to be loaded the most was bolt 44. Guaranteed fracture of these bolts would lead to fracture of the whole joint during the first semi-cycle of counterweight vibrations. 


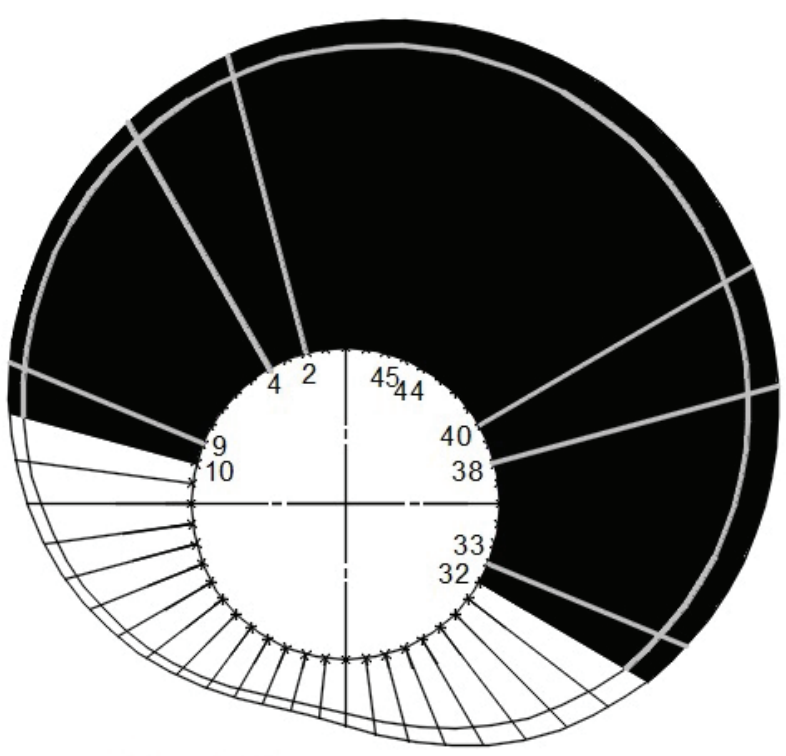

Fig. 5. Circular diagram of equivalent stresses.

Next, one needs to consider the necessity of strengthening the flange joint or developing a new design for the section which joins the slewing unit base and mast. To achieve these goals, an elemental analysis of the counterweight frame was carried out in the software environment of SolidWorks for the peak force $F_{C}$. The software allowed to build a solid model, and then to study it in the SolidWorks Simulation add-on using its finite element analysis tool [8].

\section{Finite element calculation of counterweight frame}

The counterweight frame material S235 is analogous to a domestic steel St3sp5 with the yield stress of $235 \mathrm{MPa}$. The distribution pattern for calculated normal stresses in the counterweight frame in relation to the action of the peak force $F_{C}$ is shown in Fig. 6. The maximal normal bending stress at the location of frame fastening is 283 $M P a$. Therefore, strengthening the flange joint could lead to the limit yielding state of the counterweight frame in the case of slewing unit wedging.

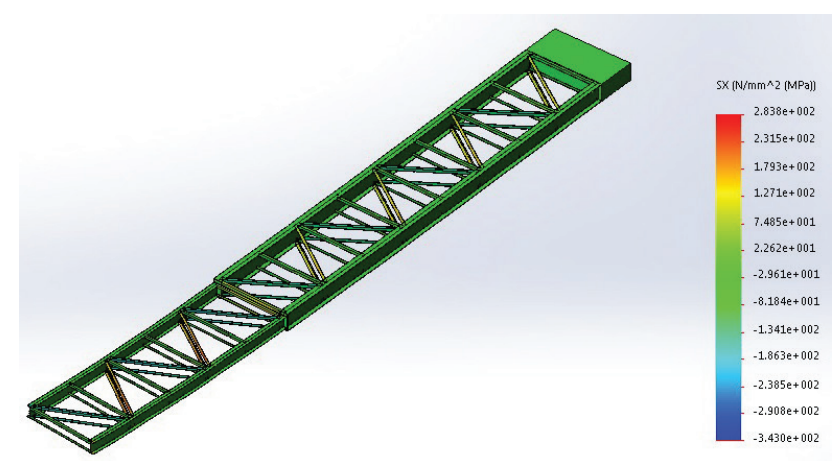

Fig. 6. Distribution of stresses on inertia force.

The calculated frame rigidity $c_{C}=903,4087 \mathrm{kN} / \mathrm{m}$ is somewhat lower than that obtained using formulas from the strength of materials. Accordingly, calculated equivalent stresses in bolts (bounded by the internal curve in Fig. 5) turned out to be lower, as well. This correction did not make any significant difference in terms of the conclusions on the cause of the accident when wedging of the slewing unit occurred. The zones of yield (bolts 9-33) and fracture (bolts 2-40) became narrower, and bolt 45 turned out to be loaded the most.

\section{Conclusion}

Locking of the slewing init of an unloaded crane which operates at its working angular velocity is an unacceptable catastrophic event. The forces of gravity and inertia of jib and counterweight masses cause, in such a bolted flange joint as the one considered here, such stresses which considerably exceed ultimate breaking loads for bolts of 10.9 strength grade.

Since practically all possible ways of strengthening the bolts have been tried out, new design solutions are needed to mount the slewing unit. For example, a ring belt to resist the severing force or spot welding of a flange joint might be introduced. But, it should be remembered that an increase in strength of a flange joint could lead to fracture of the counterweight metallic structure.

The only sure way of securing the work capacity of a crane is to adhere closely to the operating rules and eliminate emergency locking of the slewing unit.

\section{References}

1. BS EN ISO 2001:2008. Quality Management Systems - Requirements. 4th. Ed. (Management Centre, Brussels, 2008)

2. S.A. Kazak, Bridge Crane Dynamics (Mashinostroenie, Moscow, 1968)

3. D.V. MacCollum, Crane hazards and their prevention (ASSE, USA, 2005)

4. H. Ouyang, Mech. Syst. Signal Pr., 25, 2039 (2011)

5. V.I. Smirnov, Course on Higher Mathematics (Nauka, Moscow 2, 1974)

6. H.A. Rahmatulin, Ju.A. Dem'janov, Strength under Intensive Short-term Loads (Izd. fizikomatematicheskoj literatury, Moscow, 1961)

7. I.G. Emel'yanov, V.I. Mironov, A.V. Kuznetsov, AIP Conf. Proc., 1785, 040014 (2016)

8. A.A. Aljamovskij, A.A. Sobachkin, E.V. Odincova, etc., Computer-aided Design in SolidWorks for Engineering Purposes (BHV-Peterburg, Saint Petersburg, 2005, 800 p.) 